Original Paper http://ajol.info/index.php/ijbcs http://indexmedicus.afro.who.int

\title{
Diversité floristique des dépendances vertes périurbaines de Brazzaville (Congo) menacées de dégradation
}

\section{Mat-Sheridan MIKOUNGUI GOMO ${ }^{1 *}$, Donatien N'ZALA ${ }^{1}$ et Saint Fédriche NDZAI ${ }^{1}$}

Laboratoire de Géomatique et d'Écologie Tropicale Appliquée (LGETA), École Nationale Supérieure d'Agronomie et de Foresterie (ENSAF), Université Marien N'GOUABI ; BP. 69, Brazzaville, Congo.

*Auteur correspondant, E-mail: matsheridan2013@gmail.com; Tél : +242069198970

\section{RESUME}

A Brazzaville, les dépendances vertes jouent un rôle important sur le bien-être et le cadre de vie des citadins. Cependant elles sont menacées de dégradation à cause d'une urbanisation mal contrôlée. Cette étude a été menée pour identifier et caractériser la biodiversité végétale de ces milieux. A cet effet, des relevés floristiques ont été réalisés au niveau de deux types de phytocénoses «dégradée » et «semi intacte » dans deux sites des quartiers périphériques de Ngamakosso et Sadelmi suivant un dispositif de 17 parcelles (30 m x $20 \mathrm{~m}$ ). Au total, 205 espèces végétales reparties en 160 genres et 65 familles ont été inventoriées. La présence/abondance de certaines espèces et familles (Asteraceae, Rubiaceae, Poaceae) associée avec l'appréciable proportion des nanophanérophytes, révèle le caractère arbustif et perturbé de cette végétation sur des sols sableux et pauvres, favorables aux érosions. La conservation et la reconstitution des phytocénoses étudiées sont assurées grâce à des espèces endémiques, guinéo-congolaises et pantropicales, à très large distribution. Les connaissances acquises de cette étude suggèrent d'intégrer la problématique de l'aménagement des espaces végétalisés urbains dans les politiques de développement durable de la ville.

(C) 2020 International Formulae Group. All rights reserved.

Mots clés : Richesse floristique, biodiversité urbaine, phytocénose, conservation, reconstitution.

\section{Floristic diversity of peri-urban green dependencies in Brazzaville (Congo) threatened by degradation}

\begin{abstract}
In Brazzaville, green dependencies have an important role in the well-being and living environment of city dwellers. However, they are threatened with degradation due to poorly controlled urbanization. This study was conducted to identify and characterize the plant biodiversity of these habitats. For this purpose, floristic surveys were carried out at the level of two kinds of "degraded" and "semi intact" phytocenosis at two sites in the outlying areas of Ngamakosso and Sadelmi following a scheme of 17 plots (30 m x $20 \mathrm{~m}$ ). In total, 205 plant species distributed into 160 genera and 65 families were inventoried. The presence/abundance of certain species and families (Asteraceae, Rubiaceae, Poaceae) associated with the significant proportion of nanophanerophytes,
\end{abstract}


revealed the shrubby and disturbed character of this vegetation on sandy, poor and erosive soils. The conservation and reconstitution of the studied phytocenosis are ensured by endemic, Guineo-Congolese and pantropical species, with very wide distribution. The knowledge acquired from this study suggests integrating the issue of vegetative landscaping into the city's sustainable development policies.

(C) 2020 International Formulae Group. All rights reserved.

Keywords: Floristic richness, urban biodiversity, phytocenosis, conservation, reconstitution

\section{INTRODUCTION}

La biodiversité est actuellement un enjeu majeur de la recherche en écologie, à la fois sur son rôle dans les écosystèmes, son déterminisme et sa valorisation dans le domaine de la préservation de l'environnement (Breton, 2018). Depuis quelques années, la question de l'état de la biodiversité en milieu urbain et sa gestion est devenue capitale (écologie urbaine, développement durable, ...). La capacité des aires urbaines à héberger une biodiversité variée et fonctionnelle fait l'objet de nombreuses études, dont l'objectif majeur est d'identifier un moyen innovant de conserver et promouvoir la biodiversité (Savard et al., 2000). La littérature scientifique apporte des preuves solides attestant que les parcs constituent des hotspots («points chauds ») de biodiversité en milieu urbain (Cornelis et Hermy, 2004). Comme dans toutes zones urbaines, les ressources végétales jouent un rôle important dans la protection des sites, la lutte contre le réchauffement climatique et la satisfaction des besoins des populations (Kimpouni et al., 2013 ; Laïlle et al., 2013).

La végétation de Brazzaville (jadis appelée «la verte ») se compose actuellement d'arbres alignés, de cour, d'espaces verts (N'zala et Miankodila, 2002), de plantations forestières, de reliques de forêts naturelles et des dépendances vertes naturelles. Celles-ci sont des espaces végétalisés d'entre deux blocs de bâtis, des reliques de savane, localisées sur des terrains plats, en pente, versants de collines surtout sur les flancs des bassins versants. Ces ressources connaissent depuis plus d'une décennie, un processus de dégradation significative suite à une urbanisation non contrôlée et les multiples interventions de l'homme (Goma Boumba, 2009). La conséquence est souvent la dégradation de la végétation, puis l'appauvrissement des terres et l'érosion des sols. Les études antérieures relatives à la phytodiversité des forêts urbaines et périurbaines de Brazzaville (N'zala, 1980 ; Kimpouni et al., 2013; Koubouana et al., 2016), montrent toutes, leur état de dégradation avancé ayant conduit à la réduction de la taille, sinon à la disparition de certaines forêts. Les dépendances vertes quant à elles ne sont pas étudiées. Elles sont également menacées de dégradation et de disparition quoique le rôle de la végétation dans la protection des sols soit bien connu. Leur connaissance, du point de vue scientifique, et leur préservation au plan environnemental, s'avèrent ainsi nécessaires et constituent une préoccupation majeure.

L'objectif de cette étude a été d'affiner la connaissance de la diversité floristique des dépendances vertes urbaines en général et particulièrement celles de Brazzaville. Elle concerne les zones périphériques des quartiers de Ngamakosso (Talangaii) et de Sadelmi (Mfilou Ngamaba) en prise à des érosions pluviales. Elle vise l'amélioration des connaissances en rapport avec l'hypothèse selon laquelle les dépendances vertes urbaines sont riches en biodiversité. L'étude permettra aussi de mieux définir la place de la biodiversité dans l'aménagement du territoire et notamment des zones urbaines.

\section{MATERIEL ET METHODES}

\section{Localisation géographique de la zone et des sites d'étude}

La ville de Brazzaville, capitale de la république du Congo, est comprise entre les latitudes $4^{\circ} 6^{\prime}$ et $4^{\circ} 23^{\prime}$ Sud et les longitudes 
$15^{\circ} 5^{\prime}$ et $15^{\circ} 25^{\prime}$ Est. Elle couvre une superficie de plus $309 \mathrm{~km}^{2}$ et compte 9 arrondissements ou communes (Makélékélé, Bacongo, PotoPoto, Moungali, Ouenzé, Talangaï, Mfilou Ngamaba, Madibou et Djiri) (Figure 1). Elle a une zone périurbaine très étendue aussi bien dans sa partie nord que dans sa partie sudouest. Brazzaville se situe entre les plateaux de Cataractes au sud-ouest, de Mbé au nord-est et le fleuve Congo à l'est (Ibiassi Mahoungou et al., 2019). Le climat de Brazzaville est du type tropical humide avec une saison humide et chaude (MAM et OND) et une saison sèche et fraîche (JJAS) (Figure 2). La moyenne annuelle des précipitations varie de 1300 à $1600 \mathrm{~mm}$. La température moyenne annuelle est de l'ordre de $25^{\circ} \mathrm{C}$. L'humidité relative est toujours supérieure à $70 \%$. La géologie présente d'importantes séries essentiellement gréseuses et sableuses (Vennetier, 1977). Les sols se développent sur des matériaux sableux, pauvres en bases échangeables et très perméables. Ils sont ferralitiques fortement désaturés et appauvris (Dénis, 1970). Brazzaville est drainée par plusieurs cours d'eau dont les principaux sont : Djoué, Djiri, Tsièmé, Mfilou, Mfoa, Madoukounsiekélé. Ces cours d'eau sont des affluents du fleuve Congo. La population de Brazzaville est estimée à plus de 1500000 habitants (Ibiassi Mahoungou et al., 2019). La couverture végétale est constituée par une mosaïque savane-forêt.

Deux sites d'étude localisés dans deux communes voisines et périphériques de l'agglomération de Brazzaville ont été choisis selon leur accessibilité : Ngamakosso dans la commune de Talangaï ( $4^{\circ} 12^{\prime} 02^{\prime}$ 'S et $15^{\circ} 18^{\prime} 13^{\prime \prime}$ 'E) et Sadelmi dans la commune de Mfilou Ngamaba (413'16'’S et $\left.15^{\circ} 13^{\prime} 26^{\prime \prime} \mathrm{E}\right)$.

\section{Matériel d'étude}

Deux phytocénoses plus ou moins contiguës ont été choisies par site selon la physionomie et l'état d'évolution de leur végétation (soit quatre phytocénoses au total). L'une des phytocénoses est une ancienne zone érodée qualifiée de « dégradée » et l'autre qui n'a pas subi de perturbation apparente et connue, est dite «semi intacte».

\section{Méthodologie d'étude Collecte des données}

La démarche méthodologique adoptée est basée sur les inventaires floristiques des quatre phytocénoses. Ceux-ci ont été conduits à l'aide de la méthode des relevés de surface. Le relevé de surface a consisté à délimiter des parcelles de 30 m x 20 m $(600$ $\mathrm{m}^{2}$ ). Dix parcelles ont été installées dans le site de Ngamakosso et sept dans le site de Sadelmi, soit un ensemble de 17 parcelles dans les deux sites d'étude avec une superficie totale de $10200 \mathrm{~m}^{2}$ (soit 1,02 ha). Chaque parcelle a été subdivisée en quatre placettes de $150 \mathrm{~m}^{2}$ (15 m $\mathrm{x} 10 \mathrm{~m}$ ). Dans chacune des placettes, toutes les espèces de plantes vasculaires rencontrées ont été identifiées et notées sur une fiche de relevé portant le numéro de la parcelle. Les espèces non identifiées ont été mises en herbier pour une identification ultérieure. Par ailleurs, la nomenclature de toutes les espèces a été vérifiée en consultant l'herbier national de l'IRSEN à Brazzaville. Pour chaque phytocénose et chaque espèce inventoriée, il a été noté le type biologique en fonction du stade de développement du sujet (Raunkiaer, 1934 ; Lebrun, 1947). Les principaux types biologiques mis en évidence sont: les Phanérophytes $(\mathrm{Ph})$ : arbres, arbustes et arbrisseaux, lianes, comprenant les microphanérophytes (mph : de hauteur $2 \leq \mathrm{H}<$ $8 \mathrm{~m}$ ), les nanophanérophytes ( $\mathrm{nph}: \mathrm{H}<2 \mathrm{~m}$ ), les lianes grimpantes à vrilles (mphgrv); les Chaméphytes (Ch) : sous-arbrisseaux, les Hémicryptophytes $(\mathrm{Hc})$ : herbacées pérennes, les Géophytes (G) : plantes à tubercules, rhizomes ou bulbes et les Thérophytes (Th) : plantes annuelles. La chorologie a été également déterminée en utilisant les grandes subdivisions phytogéographiques africaines établies par de nombreux auteurs (White, 1983 ; Lubini, 2001 ; Kimpouni, 2009) : Espèces à très large distribution (Cosm : Cosmopolite, Pant : Pantropicale, Ant: Afronéotropicale [Afro-Américaine (Aa)], Pal : 
Paléotropicale); Espèces à large distribution (At : Afro-tropicale continentale, Am: Afromalgache); Espèces endémiques (GC : omniguinéo-congolaise, $\quad \mathrm{BG}$ : basguinéenne, BGC : bas-guinéo-congolaise, $\mathrm{C}$ : centro-congolaise); Espèces des zones de transition régionale; GC-Z : espèce guinéocongolaise-zambézienne ; GC-S : espèce guinéo-congolaise-soudanienne.

\section{Traitement et analyse des données}

Les données rassemblées ont été analysées suivant les indices de diversité biologique, la fréquence d'apparition dans les placettes. L'indice de Shannon $\left(\mathrm{H}^{\prime}\right)$ et l'indice d'équitabilité ou d'équirépartition de Pielou (E) et les coefficients de similitude de Sorensen et de Jaccard sont donnés par les formules mathématiques suivantes :

- l'indice de diversité de Shannon : H' $=-\Sigma$ $\left[(\mathrm{ni} / \mathrm{N}) \times \log _{2}(\mathrm{ni} / \mathrm{N})\right]$

$\mathrm{ni}=$ nombre d'individus d'une espèce dans l'échantillon ;

$\mathrm{N}=$ nombre total d'individus de toutes les espèces dans l'échantillon

- l'équitabilité de Pielou $: \mathrm{E}=\frac{\mathrm{H} \prime}{\mathrm{H} / \max }$

Il exprime la régularité, la répartition équitable des individus au sein des espèces :

$\mathrm{H}^{\prime}$ max est le logarithme du nombre total d'espèces (S). Elle représente la diversité maximale $\quad H^{\prime}{ }_{\text {max }}=\log _{2} \mathrm{~S} . \quad \mathrm{S}=$ nombre total d'espèces.

L'équitabilité varie de 0 à 1 . Elle tend vers 0 quand la quasi- totalité des effectifs est concentrée sur une espèce et vers 1 lorsque toutes les espèces ont la même abondance. Dans le cas où cet indice tend vers 1 , le milieu en question est dit équilibré.

L'indice de diversité floristique de Shannon accorde une importance aux espèces rares. La diversité floristique maximale $\left(\mathrm{H}^{\prime}\right.$ max $\left.=\log _{2} \mathrm{~s}\right)$ renseigne sur le degré de diversité que peut atteindre une phytocénose dans le cas d'une distribution équitable des individus constitutifs de sa flore. Enfin, l'indice de Pielou ou indice d'équitabilité
( $\left.\mathrm{H}^{\prime} / \mathrm{H}^{\prime} \max \right)$ indique le degré de diversité atteint par rapport au maximum possible et exprime mieux la variation intra-communauté. - le coefficient de similarité de Sorensen permet de savoir si deux relevés A et B comparés sur le plan floristique appartiennent à la même communauté végétale.

$\mathrm{K}(\%)=\frac{2 \mathrm{c}}{\mathrm{a}+\mathrm{b}} \times 100$. L'hypothèse de similitude ou ressemblance est admise, lorsque le coefficient est supérieur à $50 \%(\mathrm{Ks}>50)$. Dans le cas contraire $(\mathrm{Ks}<50)$, il n'y a pas de similitude entre les relevés floristiques concernés (Troupin, 1966).

- le coefficient de Jaccard (S) qui est plus connu se calcule de la manière suivante :

$$
\mathrm{S}(\%)=\frac{\mathrm{c}}{\mathrm{a}+\mathrm{b}-\mathrm{c}} \times 100
$$

$a=$ nombre d'espèces du relevé $A ; b=$ nombre d'espèces du relevé $B$; $c=$ nombre total des espèces communes aux relevés $\mathrm{A}$ et $\mathrm{B}$.

Le coefficient de Jaccard donne la même valeur à la présence et à l'absence, alors que celui de Sorensen donne un avantage deux fois plus important à la présence. Dans ce dernier cas la présence est plus informative que l'absence (Legendre et Legendre 1984).

Le calcul des spectres biologiques et phytogéographiques bruts s'est fait à partir de la formule utilisée par Kimpouni (2013):

$\mathrm{SB}(\%)=\frac{\text { Nombre d'espèces par famille }}{\text { Nombre total d'espèces }} \times 100$

L'examen des types biologiques permet de déterminer les stratégies adaptatives ainsi que la physionomie de la végétation. Les types phytogéographiques déterminent quant à eux, l'origine des espèces inventoriées.

\section{Mode d'expression des résultats}

Les indices de diversité floristique (Shannon et Piélou) ont été comparés au moyen de tests d'analyse de variance à un facteur (ANOVA 1). Lorsque les différences entre les moyennes des différentes phytocénoses étaient significatives $(\mathrm{p}<0,05)$, un test post-hoc (test de Duncan) a été réalisé pour classer et savoir lesquelles des phytocénoses sont différentes. 


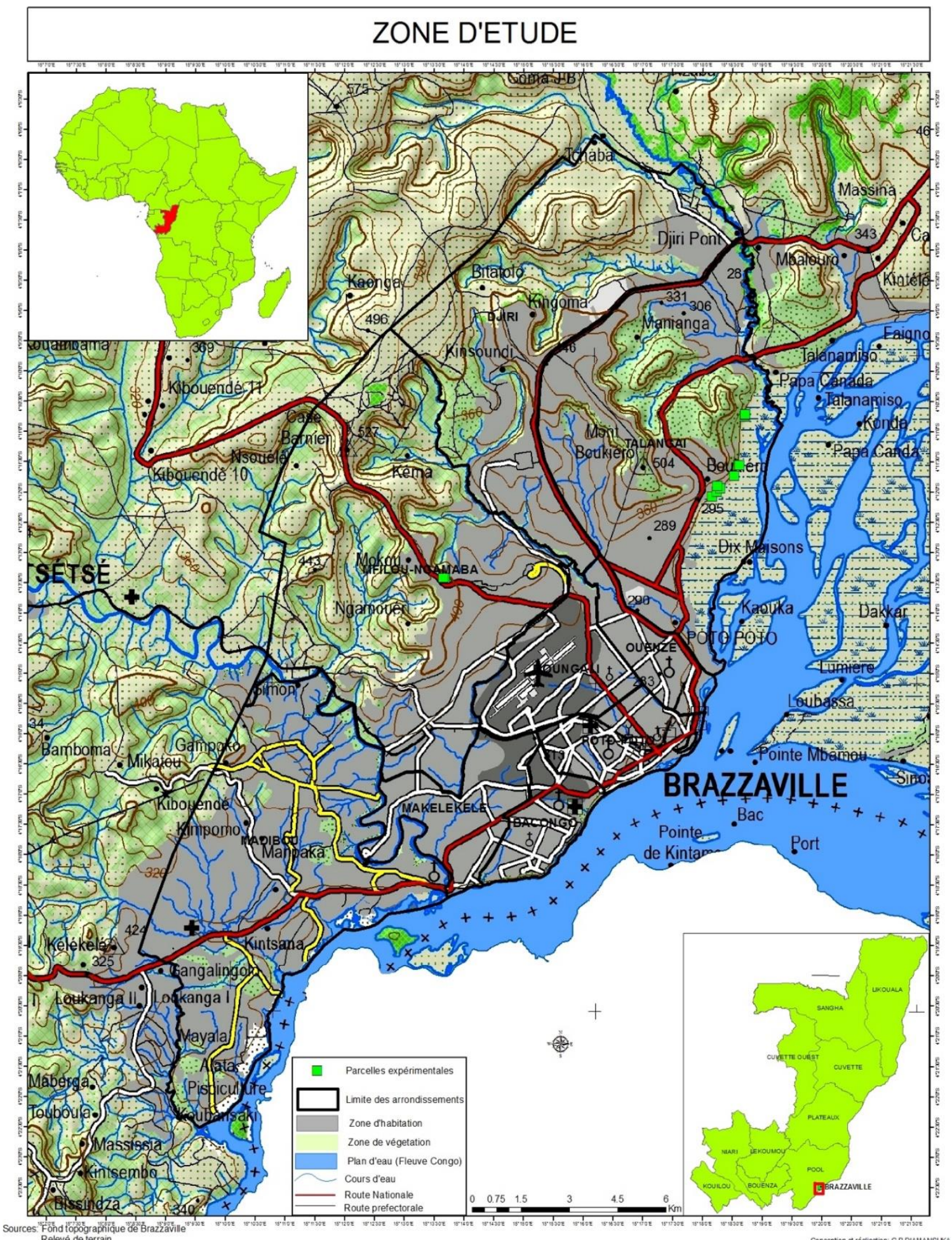

Figure 1 : Carte de situation de Brazzaville et des sites d'étude. 


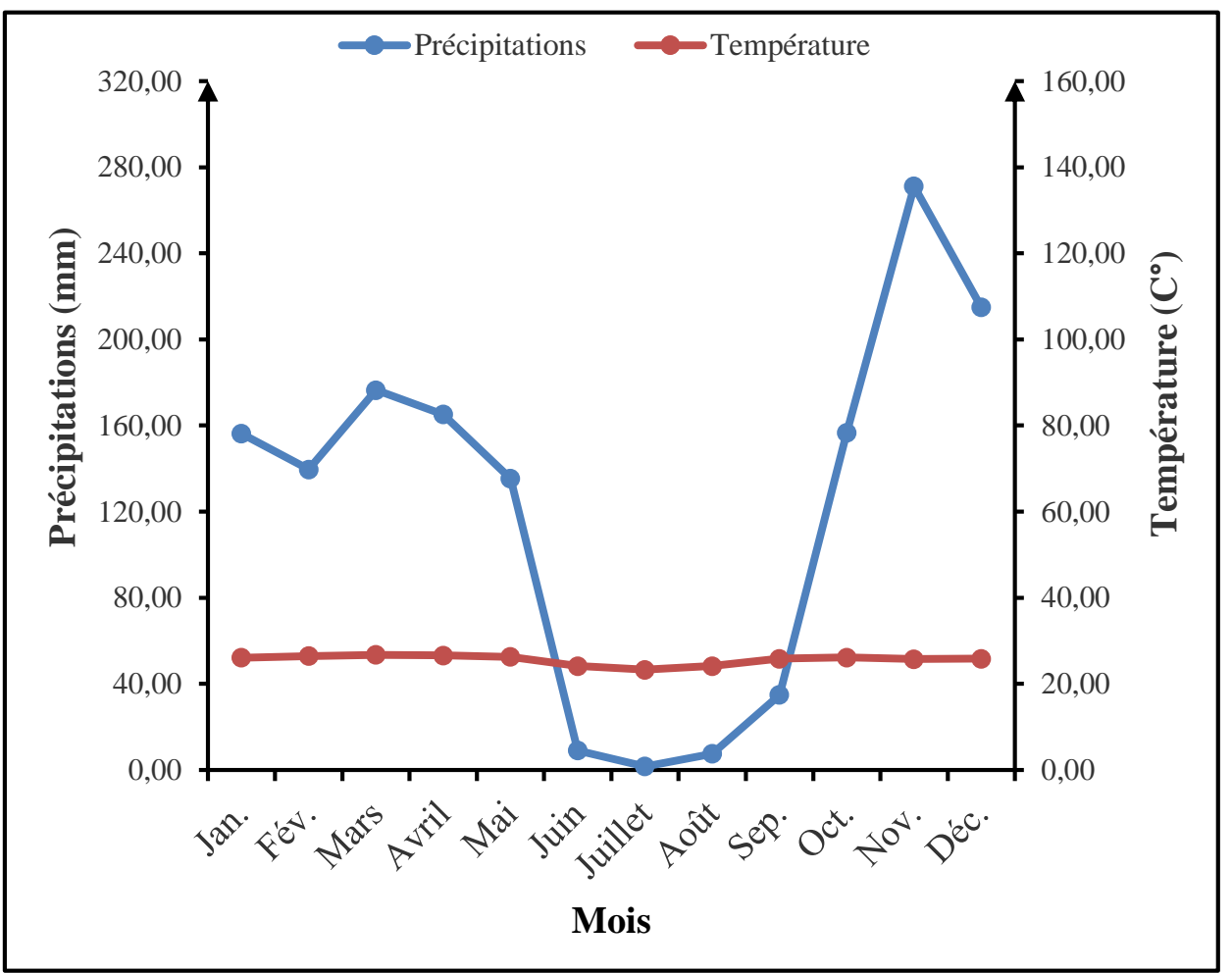

Figure 2 : Diagramme ombrothermique de Brazzaville, 2008-2018 (Source : ANAC, 2019).

\section{RESULTATS}

\section{Richesse et composition floristiques des phytocénoses étudiées}

Les inventaires ont permis de recenser 205 espèces réparties dans 160 genres et 65 familles pour l'ensemble des quatre phytocénoses (Tableau 1). La phytocénose semi intacte de Ngamakosso avec 147 espèces pour 61 familles et 120 genres est plus riche que celle de Sadelmi avec 86 espèces réparties dans 42 familles et 78 genres. L'inverse est constaté dans les phytocénoses dégradées avec 99 espèces réparties dans 46 familles et 92 genres à Sadelmi contre seulement 43 espèces distribuées dans 25 familles et 39 genres à Ngamakosso.

Selon le nombre d'espèces, la représentation des familles varie selon le site et la nature des parcelles. A Ngamakosso (Tableau 2), dans les parcelles semi intactes, les familles les plus représentées ( 5 espèces au moins) se présentent dans l'ordre suivant: Poaceae (15 espèces, soit 10,20\%), Fabaceae (Faboïdeae) (13 espèces, soit $8,84 \%)$, Rubiaceae (11 espèces, soit $7,48 \%)$, Cyperaceae (8 espèces, soit $5,44 \%)$, Euphorbiaceae (8 espèces, soit $5,44 \%$ ), Asteraceae (6 espèces, soit 4,08\%), Apocynaceae (5 espèces, soit $3,40 \%$ ) et Lamiaceae (Viticoideae) (5 espèces, soit $3,40 \%$ ). On y dénombre aussi 39 familles monospécifiques sur les 61 recensées (soit 64\% des familles). Dans les parcelles dégradées de Ngamakosso, les Poaceae (9 espèces, soit 20,93\%) et les Fabaceae (Faboïdeae) (5 espèces, soit 11,63\%) demeurent les plus représentées et sont suivies par les Asteraceae ( 3 espèces, soit $6,98 \%$ ) et dans une moindre mesure, les Acanthaceae, Cyperaceae, Lamiaceae (Viticoïdeae) et Phyllanthaceae (2 espèces, soit 4,65\%). Ici, 18 familles sur 25 sont monospécifiques (soit $72 \%$ ). A Sadelmi 
(Tableau 3), dans les parcelles semi-intactes, l'ordre des familles est le suivant : Rubiaceae (10 espèces, soit 11,63\%), Poaceae (9 espèces, soit 10,47\%), Fabaceae (Faboïdeae) (7 espèces, soit $8,14 \%$ ), Connaraceae, Cyperaceae et Euphorbiaceae (5 espèces, soit 5,81\%), Fabaceae (Mimosoïdeae) et Phyllanthaceae (3 espèces, soit $3,49 \%$ ). Soulignons que sur les 42 familles recensées dans ces parcelles, 29 sont monospécifiques (soit 69\%). Dans les parcelles dégradées, les familles les plus représentées sont les Poaceae (16 espèces, soit 17,17\%), les Fabaceae (Faboïdeae) (8 espèces, soit 8,08\%), les Asteraceae et les Rubiaceae (6 espèces, soit 6,06\%), les Euphorbiaceae (5 espèces, soit 5,05\%), les Cyperaceae, les Fabaceae (Caesalpinioideae) et les Fabaceae (Mimosoïdeae) (4 espèces, soit 4,04\%), les Connaraceae et les Phyllanthaceae ( 3 espèces, soit 3,03\%). 32 familles sur les 46 sont monospécifiques (soit $69,56 \%$ ).

En tenant compte du nombre des individus par espèce, le Tableau 4 montre, pour chaque phytocénose, les dix espèces les plus représentatives. Pour la phytocénose semi intacte de Ngamakosso, Oncoba welwitschii $(4,96 \%)$, Smilax anceps $(4,87 \%)$, Sclerocroton cornutus (4,22\%), Markhamia tomentosa $(4,20 \%)$, Hymenocardia ulmoides (3,57\%), Thomandersia butayei (3,57\%), Platysepalum vanderystii (3,55\%), Aframomum stipulatum $(3,20 \%)$, Cyperus mapaniö̈des $(2,98 \%)$ et Chromolaena odorata (2,89\%). Pour la phytocénose dégradée de Ngamakosso, on cite Cenchrus purpureus (15,09\%), Panicum maximum (12,11\%), Tithonia diversifolia $(11,89 \%)$, Chromolaena odorata $(10,13 \%)$, Panicum brazzavillense (8,81\%), Eleusine indica $(5,40 \%)$, Bambusa vulgaris $(3,63 \%)$, Urena lobata $(3,08 \%)$, Setaria sphacellata var. sphacellata $(2,42 \%)$ et Lantana camara $(2,31 \%)$. La phytocénose semi intacte de Sadelmi quant à elle présente l'ordre suivant des espèces: Hyparrhenia diplandra $(8,27 \%)$, Smilax anceps (5\%), Rhadophyllum affine (4,12\%), Aframomum stipulatum (4,05\%), Digitaria horizontalis, Pteridium aquilinum (3,60\%), Spermacoce latifolia (3,56\%),
Oncoba welwitschii (2,35\%), Pennisetum polystachion $(2,87 \%)$ et Dichapetalum librevillense $(2,25 \%)$. Pour la phytocénose dégradée de Sadelmi, Panicum maximum (12,35\%), Digitaria horizontalis $(9,69 \%)$, Tithonia diversifolia (7,17\%), Cenchrus purpureus et Mucuna flagellipes (6,14\%), Hyparrhenia diplandra (4,30\%) et Chromolaena odorata $(4,16 \%)$, sont les plus représentatives. On cite aussi Oncoba welwitschii (2,79\%), Pennisetum polystachyon $(2,79 \%)$ et Senna occidentalis (2,66\%).

Quatre groupements végétaux caractéristiques sont ainsi observés. Le premier et le deuxième issus des phytocénoses semi intactes sont définis par Oncoba welwitschii, Smilax anceps et Afromomum stipulatum associés d'une part à Sclerocroton cornutus et Hymenocardia ulmoides dans le site de Ngamakosso et d'autre part à Pteridium aquilinum, Dichapetalum librevillense et Hyparrhenia diplandra dans le site de Sadelmi. Les deux autres groupements indépendants qui apparaissent dans les phytocénoses dégradées se caractérisent par la présence d'herbacées (Poaceae: Panicum maximum et Cenchrus purpureus) et d'Asteraceae (Chromolaena odorata et Tithonia diversifolia) associés d'une part à Urena lobata, Setaria sphacellata et Lantana camara à Ngamakosso et d'autre part à Digitaria horizontalis, Pennisetum polystachion et Mucuna flagellipes à Sadelmi. Dans ce dernier groupement, persistent aussi quelques espèces des groupements semi intacts (Oncoba welwitschii et Hyparrhenia diplandra) dans des proportions encore remarquables.

\section{Spectres biologiques}

L'inventaire a permis de recenser une flore se répartissant en 11 types biologiques rassemblés en 5 grands groupes appartenant en majorité aux phanérophytes. L'analyse quantitative montre que les phanérophytes $(57,23 \%)$, sont le type dominant de la florule. Viennent ensuite les chaméphytes $(12,86 \%)$, les thérophytes $(11,95 \%)$ et les 
hémicryptophytes (10,04\%). L'importance des géophytes $(7,92 \%)$ est faible (Figure 3 ).

Les valeurs des spectres biologiques des espèces inventoriées dans les deux sites étudiés (Ngamakosso et Sadelmi) varient selon l'état d'évolution de la végétation. Dans les formations semi intactes (Tableau 5a), quel que soit le site, les phanérophytes $(63,32 \%)$ demeurent prépondérantes devant les chaméphytes $(10,04 \%)$ et les thérophytes $(9,51 \%)$. Les phanérophytes se composent des microphanérphytes (18\%), des nanophanérophytes $(26,10 \%)$ et des phanérophytes grimpantes à vrilles $(19,20 \%)$. Dans les phytocénoses dégradées (Tableau $5 \mathrm{~b}$ ), la prépondérance des phanérophytes $(51,13 \%)$ est toujours affirmée et suivent à peu près dans le même ordre les chaméphytes $(15,67 \%)$ puis les thérophytes $(14,38 \%)$ et les hémicryptophytes (11,54\%). Parmi les phanérophytes, les nanophanérophytes $(21,80 \%)$ montrent encore un taux plus élevé que les deux autres types notamment les microphanérophytes $(12,98 \%)$ et les phanérophytes grimpantes $(16,34 \%)$ quel que soit le site.

\section{Distribution phytogéographique}

L'analyse phytogéographique de la flore identifiée (Tableau 6) montre 13 types de distribution regroupés principalement en six unités supérieures dont deux sont dominantes et renferment chacune plus de $31 \%$ (soit $1 / 3$ ) de cette flore, quel que soit le site. Il s'agit des espèces à très large distribution qui sont essentiellement pantropicales $(25,99 \%)$ et des espèces endémiques qui sont en majorité guinéo-congolaises $(27,69 \%)$. Les espèces à large distribution et de zone de transition sont moins importantes avec respectivement $14,40 \%$ et $12,71 \%$.

\section{Diversité floristique}

Les indices de diversité moyens sont élevés quel que soit l'état de la phytocénose. Ils varient de 4,30 à 6,01 pour l'indice de Shannon et de 0,79 à 0,89 pour l'équitabilité de Pielou. L'analyse de variance montre des différences significatives entre les phytocénoses semi intactes et celles dégradées en ce qui concerne l'indice de Shannon (Tableau 7).

\section{Similitude entre les phytocénoses}

Les valeurs du coefficient de Sorensen (Tableau 8) montrent qu'il n'y a pas de véritable ressemblance floristique entre les différentes phytocénoses. Chaque phytocénose est bien caractérisée par un cortège floristique spécifique ayant moins d'espèces communes avec les autres. Il apparaît toutefois une affinité à Sadelmi entre la phytocénose semi intacte et celle dégradée ( $\mathrm{K}=54,84 \%)$, ce qui n'est pas le cas à Ngamakosso $(K=36,84 \%)$. Les mêmes tendances sont observées avec le coefficient de Jaccard (Tableau 8) où la valeur la plus élevée est obtenue à Sadelmi aussi entre la phytocénose semi intacte et celle dégradée $(\mathrm{S}=$ $37,78 \%)$.

Tableau 1 : Répartition taxonomique par phytocénose.

\begin{tabular}{llccc}
\hline Site & Phytocénose & Famille & Genre & Espèces \\
\hline \multirow{2}{*}{ Ngamakosso } & Semi intacte (PSINGA)* & 61 & 120 & 147 \\
\cline { 2 - 5 } & Dégradée (PDNGA) & 25 & 39 & 43 \\
\hline \multirow{2}{*}{ Sadelmi } & Semi intacte (PSISAD) & 42 & 78 & 86 \\
\cline { 2 - 5 } & Dégradée (PDSAD) & 46 & 92 & 99 \\
\hline & TOTAL & 65 & 160 & 205
\end{tabular}

Légende : PSINGA : Phytocénose semi intacte de Ngamakosso ; PDNGA : Phytocénose dégradée de Ngamakosso ; PSISAD : Phytocénose semi intacte de Sadelmi ; PDSAD : Phytocénose dégradée de Sadelmi. 
Tableau 2 : Fréquence des espèces par famille dans le site de Ngamakosso.

\begin{tabular}{|c|c|c|c|c|c|c|}
\hline & $\begin{array}{c}\text { Phytocénose semi intacte } \\
\text { (PSINGA) }\end{array}$ & $\mathrm{Ne}$ & $\%$ & $\begin{array}{c}\text { Phytocénose dégradée } \\
\text { (PDNGA) }\end{array}$ & $\mathrm{Ne}$ & $\%$ \\
\hline \multirow{10}{*}{ Famille } & Poaceae & 15 & 10,20 & Poaceae & 9 & 20,93 \\
\hline & Fabaceae ( Faboïdeae) & 13 & 8,84 & Fabaceae (Faboïdeae) & 5 & 11,63 \\
\hline & Rubiaceae & 11 & 7,48 & Asteraceae & 3 & 6,98 \\
\hline & Cyperaceae & 8 & 5,44 & Acanthaceae & 2 & 4,65 \\
\hline & Euphorbiaceae & 8 & 5,44 & Cyperaceae & 2 & 4,65 \\
\hline & Asteraceae & 6 & 4,08 & $\begin{array}{l}\text { Lamiaceae } \\
\text { (Viticoïdeae) }\end{array}$ & 2 & 4,65 \\
\hline & Lamiaceae (Viticoideae) & 5 & 3,40 & Phyllanthaceae & 2 & 4,65 \\
\hline & Araceae & 3 & 2,04 & & & \\
\hline & Connaraceae & 3 & 2,04 & & & \\
\hline & Fabaceae(Caesalpinioidea) & 3 & 2,04 & & & \\
\hline
\end{tabular}

Ne : Nombre d'espèces

Nombre de familles monospécifiques : 39 Nombre de familles monospécifiques : 18 .

Tableau 3 : Fréquence des espèces par famille dans le site de Sadelmi.

\begin{tabular}{llrrlrr}
\hline \multirow{2}{*}{ Famille } & $\begin{array}{l}\text { Phytocénose semi } \\
\text { intacte (PSISAD) }\end{array}$ & Ne & $\%$ & $\begin{array}{c}\text { Phytocénose dégradée } \\
\text { (PDSAD) }\end{array}$ & Ne & $\%$ \\
\cline { 2 - 7 } & Rubiaceae & 10 & 11,63 & Poaceae & 16 & 17,17 \\
\cline { 2 - 7 } & Poaceae & 9 & 10,47 & Fabaceae ( Faboïdeae) & 8 & 8,08 \\
\cline { 2 - 8 } & Fabaceae ( Faboï & 7 & 8,14 & Asteraceae & 6 & 6,06 \\
\hline Connaraceae & 5 & 5,81 & Rubiaceae & 6 & 6,06 \\
\hline Cyperaceae & 5 & 5,81 & Euphorbiaceae & 5 & 5,05 \\
\hline Euphorbiaceae & 5 & 5,81 & Cyperaceae & 4 & 4,04 \\
\hline Fabaceae (Mimosoïdeae) & 3 & 3,49 & Fabaceae (Caesalpinioideae) & 4 & 4,04 \\
\cline { 2 - 7 } & Phyllanthaceae & 3 & 3,49 & Fabaceae-Mimosoïdeae & 4 & 4,04 \\
\hline
\end{tabular}

$\mathrm{Ne}:$ Nombre d'espèces

Nombre de familles monospécifiques : 29

Nombre de familles monospécifiques : 32

Tableau 4 : Liste des espèces les plus représentatives par phytocénose.

\begin{tabular}{|c|c|c|c|c|c|}
\hline Espèces & Familles & PSINGA & PSISAD & PDNGA & PDSAD \\
\hline $\begin{array}{l}\text { Chromolaena odorata (L.) R. King \& } \\
\text { H. Robinson }\end{array}$ & Asteraceae & + & & + & + \\
\hline Cyperus mapaniö̈des C.B. Cl. & Cyperaceae & + & & & \\
\hline Aframomum stipulatum K. Schum & Zingiberaceae & + & + & & \\
\hline Platysepalum vanderystii De Wild. & Fabaceae(Faboïdeae) & + & & & \\
\hline Thomandersia butayei De Wild. & Thomandersiaceae & + & & & \\
\hline Hymenocardia ulmoides Oliv. & Phyllanthaceae & + & & & \\
\hline $\begin{array}{l}\text { Markhamia tomentosa (Benth.) K. } \\
\text { Schum. \& Thonn. }\end{array}$ & Bignoniaceae & + & & & \\
\hline
\end{tabular}




\begin{tabular}{|c|c|c|c|c|c|}
\hline Sclerocroton cornutus (Pax.) Kruijt & Euphorbiaceae & + & & & \\
\hline Smilax anceps Wild. & Smilacaceae & + & + & & \\
\hline Oncoba welwitschii (Oliv.) Gilg & Salicaceae & + & + & & + \\
\hline Hyparrhenia diplandra (Hack.) Stapf & Poaceae & & + & & + \\
\hline $\begin{array}{l}\text { Rhabdophyllum affine (Hook.f.) Van } \\
\text { Tiegh. }\end{array}$ & Ochnaceae & & + & & \\
\hline Digitaria horizontalis Wild. & Poaceae & & + & & + \\
\hline Pteridium aquilinum (L.) Kühn' & Dennstaedtiaceae & & + & & \\
\hline Spermacoce latifolia Aubl. & Rubiaceae & & + & & \\
\hline Pennisetum polystachion (L.) Schult & Poaceae & & + & & + \\
\hline Dichapetalum librevillense Pellegr. & Dichapetalaceae & & + & & \\
\hline $\begin{array}{l}\text { Cenchrus purpureus (Schumach.) } \\
\text { Morrone }\end{array}$ & Poaceae & & & + & + \\
\hline Panicum maximum Jacq. & Poaceae & & & + & + \\
\hline Tithonia diversifolia (Hemsl.) A. Gray. & Asteraceae & & & + & + \\
\hline Panicum brazzavillense Franch. & Poaceae & & & + & \\
\hline Eleusine indica (L.) Gaertn. & Poaceae & & & + & \\
\hline Bambusa vulgaris Schrad ex Wend & Poaceae & & & + & \\
\hline Urena lobata Linn. & Malvaceae(Malvoideae) & & & + & \\
\hline $\begin{array}{l}\text { Setaria sphacellata (Schumach) Moss } \\
\text { var. sphacellata }\end{array}$ & Poaceae & & & + & \\
\hline Lantana camara $\mathrm{L}$. & Lamiaceae(Viticoideae) & & & + & \\
\hline Mucuna flagellipes Vog. ex Benth. & Fabaceae(Faboïdeae) & & & & + \\
\hline Senna occidentalis (L.) Link. & $\begin{array}{l}\text { Fabaceae(Caesalpinioi- } \\
\text { deae) }\end{array}$ & & & & + \\
\hline
\end{tabular}

Légende : PSINGA : Phytocénose semi intacte de Ngamakosso ; PDNGA : Phytocénose dégradée de Ngamakosso ;

PSISAD : Phytocénose semi intacte de Sadelmi ; PDSAD : Phytocénose dégradée de Sadelmi

+ : présence

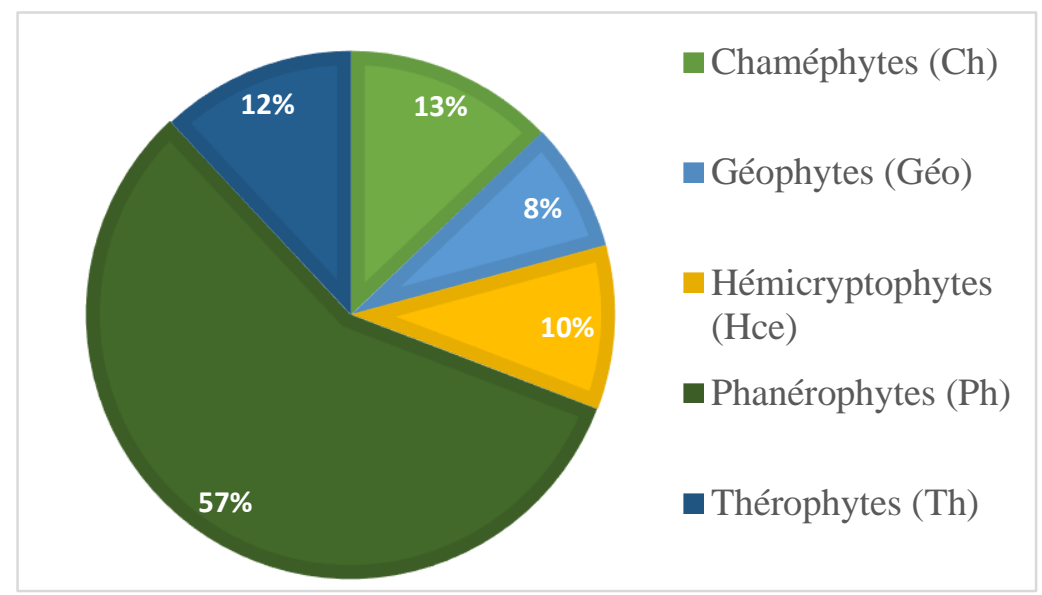

Figure 3 : Spectre biologique de la flore étudiée. 
Tableau 5 : Types et spectres biologiques des espèces inventoriées selon l'état de la phytocénose.

5a : Phytocénoses semi intactes.

\begin{tabular}{|c|c|c|c|c|c|c|c|c|}
\hline & Site & \multicolumn{3}{|c|}{ NGAMAKOSSO } & \multicolumn{3}{|c|}{ SADELMI } & \multirow{2}{*}{$\begin{array}{l}\text { Moyenne } \\
\text { générale }\end{array}$} \\
\hline \multicolumn{2}{|c|}{ Types biologiques } & $\mathrm{Nb}^{*}$ & SB* & $\%)$ & $\mathrm{Nb}$ & SB & & \\
\hline \multirow[t]{2}{*}{ Chaméphytes } & Chd & 14 & 9,52 & 14,28 & 5 & 5,81 & 5,81 & 10,04 \\
\hline & Chpr & 7 & 4,76 & & - & - & & \\
\hline \multirow[t]{2}{*}{ Géophytes } & $\mathrm{Gr}$ & 13 & 8,85 & \multirow[t]{2}{*}{10,21} & 6 & 6,97 & 6,97 & 8,59 \\
\hline & Gt & 2 & 1,36 & & - & - & & \\
\hline Hémicrptophytes & $\mathrm{Hc}$ & 8 & 5,44 & 5,44 & 10 & 11,63 & 11,63 & 8,53 \\
\hline \multirow[t]{3}{*}{ Phanérophytes } & $\mathrm{Mph}$ & 29 & 19,73 & \multirow[t]{3}{*}{59,19} & 14 & 16,28 & 67,45 & 63,32 \\
\hline & $\mathrm{Nph}$ & 34 & 23,13 & & 25 & 29,07 & & \\
\hline & Phgrv & 24 & 16,33 & & 19 & 22,10 & & \\
\hline \multirow[t]{3}{*}{ Thérophytes } & Thce & 3 & 2,04 & \multirow[t]{3}{*}{10,88} & - & - & 8,14 & 9,51 \\
\hline & Thd & 6 & 4,08 & & 4 & 4,65 & & \\
\hline & Thpr & 7 & 4,76 & & 3 & 3,49 & & \\
\hline \multicolumn{2}{|l|}{ TOTAL } & 147 & \multicolumn{2}{|c|}{100} & 86 & \multicolumn{2}{|c|}{100} & 100 \\
\hline
\end{tabular}

5b : Phytocénoses dégradées.

\begin{tabular}{|c|c|c|c|c|c|c|c|c|}
\hline & Site & \multicolumn{3}{|c|}{ NGAMAKOSSO } & \multicolumn{3}{|c|}{ SADELMI } & \multirow{2}{*}{$\begin{array}{l}\text { Moyenne } \\
\text { générale }\end{array}$} \\
\hline \multicolumn{2}{|c|}{ Types biologiques } & $\mathrm{Nb}$ & & & Nb & SB & & \\
\hline \multirow[t]{2}{*}{ Chaméphytes } & Chd & 5 & 11,63 & \multirow[t]{2}{*}{20,93} & 6 & 6,25 & \multirow[t]{2}{*}{10,42} & 15,67 \\
\hline & Chpr & 4 & 9,30 & & 4 & 4,17 & & \\
\hline \multirow[t]{2}{*}{ Géophytes } & $\mathrm{Gr}$ & 3 & 6,98 & \multirow[t]{2}{*}{9,31} & 4 & 4,17 & \multirow[t]{2}{*}{5,21} & 7,26 \\
\hline & Gt & 1 & 2,33 & & 1 & 1,04 & & \\
\hline Hémicrptophytes & $\mathrm{Hc}$ & 5 & 11,63 & 11,63 & 11 & 11,46 & 11,46 & 11,54 \\
\hline \multirow[t]{3}{*}{ Phanérophytes } & $\mathrm{Mph}$ & 4 & 9,30 & \multirow[t]{3}{*}{41,85} & 16 & 16,67 & \multirow[t]{3}{*}{60,42} & \multirow[t]{3}{*}{51,13} \\
\hline & $\mathrm{Nph}$ & 8 & 18,60 & & 24 & 25,00 & & \\
\hline & Phgrv & 6 & 13,94 & & 18 & 18,75 & & \\
\hline \multirow[t]{3}{*}{ Thérophytes } & Thce & 1 & 2,33 & \multirow[t]{3}{*}{16,28} & 2 & 2,08 & \multirow[t]{3}{*}{12,49} & \multirow[t]{3}{*}{14,38} \\
\hline & Thd & 3 & 6,98 & & 8 & 8,33 & & \\
\hline & Thpr & 3 & 6,98 & & 2 & 2,08 & & \\
\hline TOTAL & & 43 & \multicolumn{2}{|c|}{100} & 96 & \multicolumn{2}{|c|}{100} & 100 \\
\hline
\end{tabular}

Légende: Chd: Chaméphytes dressés ; Chpr: chaméphytes prostrés

Gr: Géophytes rampants; Gt: Géophytes tubéreux ; Hc: Hémicryptophytes;

mph: microphanérophytes; nph: nanophanérophytes; phgrv: phanérophytes grimpantes à vrilles

Thce: Thérophytes cespiteux; Thd: Thérophytes dressés ; Thpr : Thérophytes prostrés

$\mathrm{Nb}$ : Nombre d'espèces $\quad \mathrm{SB}:$ Spectre brut 
Tableau 6 : Distribution phytogéographique des espèces inventoriées par site d'étude.

\begin{tabular}{|c|c|c|c|c|c|c|c|c|}
\hline \multirow{2}{*}{\multicolumn{2}{|c|}{$\begin{array}{c}\text { Site } \\
\text { Origine des espèces }\end{array}$}} & \multicolumn{3}{|c|}{ NGAMAKOSSO } & \multicolumn{3}{|c|}{ SADELMI } & \multirow{2}{*}{$\begin{array}{l}\text { SB } \\
(\%)\end{array}$} \\
\hline & & \multirow{2}{*}{$\begin{array}{r}\mathbf{N b}^{*} \\
4\end{array}$} & \multicolumn{2}{|c|}{ Pourcentage (\%) } & \multirow{2}{*}{$\begin{array}{l}\mathrm{Nb} \\
2\end{array}$} & \multicolumn{2}{|c|}{ Pourcentage (\%) } & \\
\hline \multirow{4}{*}{$\begin{array}{l}\text { A très large } \\
\text { distribution }\end{array}$} & $\mathrm{Aa}$ & & 2,26 & 33,90 & & 1,13 & 31,64 & 32,77 \\
\hline & Cosm & 3 & 1,69 & & 2 & 1,13 & & \\
\hline & $\mathrm{Pal}$ & 7 & 3,96 & & 6 & 3,39 & & \\
\hline & Pant & 46 & 25,99 & & 46 & 25,99 & & \\
\hline \multirow{2}{*}{$\begin{array}{l}\text { A large } \\
\text { distribution }\end{array}$} & $\mathrm{Am}$ & 2 & 1,13 & 16,95 & 2 & 1,13 & 11,86 & 14,40 \\
\hline & At & 28 & 15,82 & & 19 & 10,73 & & \\
\hline \multirow[t]{3}{*}{ Endémique } & $\mathrm{C}$ & 5 & 2,82 & 32,77 & 10 & 5,65 & 40,68 & 36,72 \\
\hline & $\mathrm{GC}$ & 44 & 24,87 & & 54 & 30,51 & & \\
\hline & BGC & 9 & 5,08 & & 8 & 4,52 & & \\
\hline \multirow{2}{*}{$\begin{array}{l}\text { Zone de } \\
\text { transition }\end{array}$} & GC-Z & 20 & 11,30 & 12,43 & 18 & 10,18 & 13,00 & 12,71 \\
\hline & GC-S & 2 & 1,13 & & 5 & 2,82 & & \\
\hline \multirow[t]{3}{*}{ Autres } & $\mathrm{SZ}$ & 1 & 0,56 & 0,56 & - & - & - & 0,56 \\
\hline & $\mathrm{Z}$ & 6 & 3,39 & 3,39 & 5 & 2,82 & 2,82 & 3,10 \\
\hline & TOTAL & 177 & 10 & & 177 & 10 & & 100 \\
\hline
\end{tabular}

* Le nombre $(\mathrm{Nb})$ est la somme des espèces des parcelles en zone intacte et en zone dégradée dans chaque site ; $\mathrm{SB}=$ spectre brut.

Tableau 7 : Indices de diversité de Shannon et de Pielou calculés par phytocénose.

\begin{tabular}{rcc}
\hline Phytocénose & Indice de Shannon & Indice d'équitabilité de Pielou \\
\hline PSINGA & $6,01 \mathrm{a}^{*}$ & $0,84 \mathrm{a}$ \\
\hline PSISAD & $5,77 \mathrm{a}$ & $0,89 \mathrm{a}$ \\
\hline PDNGA & $4,30 \mathrm{c}$ & $0,79 \mathrm{a}$ \\
\hline PDSAD & $5,23 \mathrm{~d}$ & $0,79 \mathrm{a}$
\end{tabular}

Légende : PSINGA : Phytocénose semi intacte de Ngamakosso ; PDNGA : Phytocénose dégradée de Ngamakosso ; PSISAD : Phytocénose semi intacte de Sadelmi ; PDSAD : Phytocénose dégradée de Sadelmi

* Les valeurs non suivies par une même lettre dans la même colonne sont significativement différentes au seuil de $\mathrm{p}<0,05$ selon le test de Duncan

Tableau 8 : Coefficients de similitude de Sorensen et de Jaccard.

\begin{tabular}{lcc}
\hline Phytocénose & $\begin{array}{c}\text { Indice de Sorensen } \\
\mathbf{K}(\boldsymbol{\%})\end{array}$ & $\begin{array}{c}\text { Indice de Jaccard } \\
\mathbf{S}(\boldsymbol{\%})\end{array}$ \\
\hline PSINGA-PSISAD & 46,15 & 30,00 \\
\hline PDNGA-PDSAD & 38,03 & 23,48 \\
\hline PDNGA-PSINGA & 36,84 & 28,58 \\
\hline PDSAD-PSISAD & $\mathbf{5 4 , 8 4}$ & $\mathbf{3 7 , 7 8}$
\end{tabular}

Légende : PSINGA : Phytocénose semi intacte de Ngamakosso ; PDNGA : Phytocénose dégradée de Ngamakosso ; PSISAD : Phytocénose semi intacte de Sadelmi ;

PDSAD : Phytocénose dégradée de Sadelmi. 


\section{DISCUSSION}

Les valeurs de la richesse floristique de cette étude varient selon les sites et l'état de la végétation. La différence intersites pourrait s'expliquer par l'action anthropique sur la végétation (désherbage, brûlis, prélèvement des plantes, etc.) résultant de l'urbanisation sauvage et nul doute par les conditions pédoclimatiques. Pour Yoka et al. (2010), le climat et le sol jouent un rôle essentiel dans la composition et la structure floristiques Selon Wezel et Schlecht (2004), plus que le facteur climatique, c'est la pression anthropique qui parachève la disparition des espèces donc de la biodiversité après que les changements climatiques auront entraîné la perte de densité des espèces endémiques.

La comparaison de la végétation dans les parcelles semi intactes et dégradées permet de voir les changements apparus dans la végétation phanérogamique ; lesquels affectent les compositions floristiques ainsi que les proportions entre les espèces. Quatre groupements végétaux sont mis en évidence, caractérisés chacun par deux ou trois espèces. Ils correspondent bien aux quatre phytocénoses étudiées, ce qui confirme la spécificité de chacune d'elles. Dans les phytocénoses semi intactes, les espèces les plus abondantes sont constituées beaucoup plus d'arbustes et de lianes (Oncoba welwitschii, Smilax anceps, Sapium cornutus, Markhamia tomentosa) et de Poaceae (Hyparrhenia diplandra). Leur composition floristique se rapproche de celle des savanes arbustives telles que décrites par Makany (1976), Schwartz et al. (1995), Lubalega (2016). Ces savanes sur sols sableux sont en général dominées par une ou deux, plus rarement trois graminées, le couvert ligneux est faible. Toujours selon ces auteurs, le contact avec la forêt est toujours brutal et la lisière forestière occupe une frange de quelques mètres, parfois ceinturée par une zone herbacée dominée par différentes espèces (fougères, Aframomum, ...). Les phytocénoses dégradées sont caractérisées par la présence des Poaceae (Panicum maximum, Cenchrus purpureus, Digitaria horizontalis) et des Asteraceae (Chromolaena odorata, Tithonia diversifolia). Elles se distinguent néanmoins entre elles et des phytocénoses semi intactes. Cependant à Sadelmi, certaines espèces des phytocénoses semi intactes se rencontrent dans les phytocénoses dégradées (Oncoba welwitschii, Hyparrhenia diplandra, Pennisetum polystachyon, ...). Elles y régressent seulement, sans pour autant disparaitre au profit d'espèces adventices graminéennes (Panicum maximum, Digitaria horizontalis, Cenchrus purpurens) qui referment progressivement le tapis végétal. Elles seraient des reliques de la végétation ancienne ou proviendraient de la régénération de ces espèces à partir des stocks de semences du sol. Ce qui induit un état de conservation ou de reconstitution de la phytocénose. A Sadelmi, cette étape de la reconstitution se compose surtout d'anciennes espèces. A Ngamakosso par contre, les espèces anciennes disparaissent presque totalement au profit des espèces nouvelles, typiques des zones dégradées ou anthropisées. Elles appartiennent principalement aux Asteraceae (Chromolaena odorata, Tithonia diversifolia), Poaceae (Cenchrus purpureus, Panicum maximum, Panicum brazzavillense, Eleusine indica, Bambusa vulgaris, Setaria sphacellata, etc.) et Malvaceae (Malvoideae) (Urena lobata). Ici, la reconstitution des phytocénoses dégradées est faite surtout par de nouvelles espèces qui s'y introduisent. Ainsi, les changements de la végétation sont apparus plus radicaux à Ngamakosso qu'à Sadelmi.

L'analyse phytogéographique montre une prédominance des espèces endémiques et des espèces à très large distribution (dans des proportions équivalentes quel que soit le site). L'endémisme est un indicateur important dans l'évaluation de la biodiversité. Une espèce est dite endémique si son aire de répartition est limitée à une région donnée (Ramade, 2002 ; Tuo et al., 2017). Au niveau de la chorologie locale, la région de Brazzaville fait partie intégrante du secteur de transition bas-guinéocongolais-zambézien et du district phytogéographique de la Léfini (Descoings, 1975 ; Kimpouni et al., 1992 ; Miabangana et al., 2015). Ce domaine est caractérisé par une végétation classée dans le groupe de savane arbustive d'après la terminologie de Lebrun et 
Gilbert (1954) sur terre ferme. Les espèces endémiques plus adaptées aux conditions locales sont plus enclin à la régénération et participent davantage à la reconstitution des zones dégradées. La présence d'espèces plurirégionales témoigne d'une végétation perturbée; la forte proportion des espèces à très large distribution est un indice de perturbation et indique que la flore perd de sa spécificité (Sinsin, 2001 ; Tiokeng et al., 2015).

L'analyse du spectre des types biologiques quant à elle montre une dominance des espèces phanérophytes suivies des chaméphytes et thérophytes, tandis que les hémicryptophytes et les géophytes sont peu nombreux. La prédominance des phanérophytes indique que ces espèces s'adaptent mieux aux conditions du milieu tandis-que la proportion élevée des nanophanérophytes dénote l'importance des espèces arbustives dans la végétation de la zone d'étude (Makany, 1976; Schwartz et al., 1995). Il en est de même des chaméphytes qui supportent plus ou moins bien les courtes sécheresses. Elles font parties aussi des principales plantes caractéristiques propres à la végétation des savanes (Schwartz et al., 1995). Les thérophytes quant à elles sont classiquement considérés comme étant particulièrement adaptés aux forts régimes de perturbation et aux conditions de stress induites par les actions anthropiques. En effet, les thérophytes bouclent leur cycle pendant la saison des pluies et passent la saison sèche à l'état de graines, durant laquelle se réalisent des actions anthropiques néfastes (feux, désherbage, ...) (Morou, 2010).

L'analyse de la diversité floristique par les indices de diversité de Shannon et d'équitabilité de Pielou authentifie l'existence des quatre groupements végétaux. Les valeurs élevées de diversité des espèces dans les groupements seraient dues, d'une part à l'endémisme des principales espèces inventoriées qui disposeraient aussi de réserves importantes de graines dans le sol, et d'autre part à l'introduction des espèces à très large distribution. Toutes ces espèces sont généralement à forte potentialité de régénération (Kimpouni et al., 2013).
Les faibles valeurs des indices de similitude confirment les différences de composition floristique entre les phytocénoses. Ce qui corrobore aussi l'existence de quatre groupements végétaux mis en évidence par l'indice de diversité de Shannon. La similarité entre les deux groupements de Sadelmi indique, soit que les actions anthropiques sont moins intenses en ce lieu (d'où la présence dans le groupement dégradé de nombreuses espèces reliques de la végétation ancienne), soit que la conservation / reconstitution est assurée avant tout par les espèces anciennes ou endémiques (adaptées aux conditions locales et disposant donc de réserves de graines dans le sol).

\section{Conclusion}

L'intérêt de cette étude a été d'évaluer la composition et la diversité floristiques des dépendances vertes périurbaines de Brazzaville menacées de dégradation. La phytodiversité est aussi importante et les espèces sont équitablement réparties dans les phytocénoses étudiées que dans les autres formations savanicoles du Congo. Elle est relative à la prépondérance des espèces endémiques adaptées aux conditions locales et capables de régénération. A Sadelmi, la similitude entre la phytocénose semi intacte et celle dégradée, indique, de facto, la tendance à la conservation ou à la reconstitution de ces phytocénoses par des anciennes espèces. De façon générale, la végétation étudiée présente deux faciès : un en cours de conservation plus ou moins réussie et l'autre dégradé mais susceptible de reconstitution. L'étude confirme l'idée selon laquelle les dépendances vertes urbaines sont riches en biodiversité et révèle les tendances d'une part à la conservation et d'autre part à la reconstitution de ces phytocénoses. Les connaissances acquises concernant cette végétation pourront aider à la prise des décisions relatives à l'aménagement durable du territoire urbain.

\section{CONFLIT D'INTERETS}

Les auteurs de ce manuscrit déclarent qu'il n'y a aucun conflit d'intérêts entre eux. 


\section{CONTRIBUTIONS DES AUTEURS}

MSM et SFN ont réalisé la collecte et le traitement des données. MSM et DN ont contribué à l'interprétation des résultats et à la rédaction du manuscrit. Tous les auteurs ont approuvé la version finale du manuscrit.

\section{REMERCIEMENTS}

Les auteurs témoignent leur gratitude à M. Chérubin OUISSIKA pour son assistance technique dans la cartographie, de même qu'aux relecteurs anonymes qui ont contribué à améliorer ce travail.

\section{REFERENCES}

Breton JM. 2018. Biodiversité, écologie et droit, Études caribéennes [En ligne], DOI :

https://doi.org/10.4000/etudescaribeenne s.13001

Cornelis J, Hermy M. 2004. Biodiversity relationships in urban and suburban parks in Flanders. Landscape and Urban Planning, $\quad 69$ : $385-401$. DOI: 10.1016/j.landurbplan.2003.10.038

Denis B. 1970. Les Sols de la Région de Brazzaville. ORSTOM : Brazzaville ; 3989.

Descoings B. 1975. Les grandes régions naturelles du Congo. Candollea, 30 : 91120.

DOI : http://dx.doi.org/10.4314/1jbcs.V10i2.13 papier original.

Goma Boumba HB. 2009. Cartographie et suivi diachronique du couvert végétal de Brazzaville (approche méthodologique et analyse bibliographique). Mémoire de DEA, FLASH, Université Marien Ngouabi, Brazzaville, 72 p.

Ibiassi Mahoungou G, Maniaka FW, Goma Boumba HB. 2018. Spatialisation des températures de surface et caractérisation de l'ilot de chaleur urbain à Brazzaville. Revue de Géographie Tropicale et d'Environnement, 2 : 90100.

Kimpouni V, Mbou P, Gakosso G, Motom M. 2013. Biodiversité floristique du sousbois et régénération naturelle de la forêt de la Patte d'Oie de Brazzaville, Congo.
Int. J. Biol. Chem. Sci., 7(3): 1255-1270. DOI :10.4314/Ijbcs.V7i3.31.

Kimpouni V. 2009. Contribution à l'inventaire et à l'analyse de la flore ligneuse du plateau des cataractes (CongoBrazzaville). Acta Bot. Gallica, 156 (2) : 233-244.

Kimpouni V, Lejoly J, Lisowski S. 1992. Les Eriocaulaceae du Congo. Fragm. Flor. Geobot., 37(1): 127-145. DOI: http://dx.doi.org./10.1111/j.10958339.2009.00996.x.

Koubouana F, Ifo SA, Loupet MLB, Ndinga E. 2016. Diversité floristique et dynamique de reconstitution de la forêt du Parc Zoologique sous plantations à eucalyptus à Brazzaville, Congo. Int. J. Biol. Chem. Sci., 10(2): 609-619. DOI : 10.4314/Ijbcs.v10i2.13.

Laille P, Provendier D, Colson F, Salanié J. 2013. Les bienfaits du végétal en ville : étude des travaux scientifiques et méthode d'analyse. Plante \& Cité, Angers, $31 \mathrm{p}$.

Lebrun J, Gilbert G. 1954. Une classification écologique des forêts du Congo. Publ. INEAC, $\quad 63: \quad 89 \quad$ p. https://lib.ugent.be/catalog/rug01:001697 708

Lebrun J. 1947. La Végétation de la plaine alluviale au Sud du Lac Edouard. Bruxelles (vol. 22). Inst. Parc Nat : Congo-Belge ; $800 \mathrm{p}$.

Legendre L, Legendre P. 1984. Écologie Numérique, tome 2: - La Structure des Données Ecologiques (2ème édn). Masson : Paris ; $335 \mathrm{p}$.

Lubalega T. 2016. Évolution naturelle des savanes mises en défens à Ibi-village, sur le plateau des Batéké, en République Démocratique du Congo. Thèse en cotutelle des Sciences Forestières de l'Université Laval et l'Université de Kinshasa, Université Laval, Québec. 151 p.

Lubini Ayingweu C. 2001. Analyse phytogéographique de la flore forestière du secteur du Kasaï au Congo-Kinshasa. Syst. Geogr. Pl., 71(2): 859-872. DOI: 


\section{$10.2307 / 3668724$}

https://www.jstor.org/stable/3668724

Makany L. 1976. Végétation des plateaux Teke (Congo). Coll. Travaux Université de Brazzaville, Congo, $301 \mathrm{p}$

Miabangana, ES, Lubini Ayingweu C. 2015. Analyse floristique et phytogéographique de la végétation de l'île Loufézou à Brazzaville (République du Congo). GeoEco-Trop., 39 (1) : 55-66.

Morou B. 2010. Impacts de l'occupation des sols sur l'habitat de la girafe au Niger et enjeux pour la sauvegarde du dernier troupeau de girafes de l'Afrique de l'Ouest. Thèse de Doctorat unique en Biologie appliquée, Faculté des Sciences et Techniques, Université Abdou Moumouni de Niamey, Niger, 231 p.

N'zala D, Miankodila P. 2002. Arbres et espaces verts à Brazzaville (Congo). Bois et Forêts des Tropiques, 272 (2): 88-92. DOI: 10.19182/BFT2002. 272.A20158.

N'zala D. 1980. Dégradation de l'environnement forestier de Brazzaville. Mémoire Ingénieur. IDR, Université Marien Ngouabi, Brazzaville, 70 p.

Ramade F. 2002. Dictionnaire Encyclopédique de l'Ecologie et des Sciences de l'Environnement. Dunod : Paris ; 1075 p.

Raunkiaer C. 1934. The Life's Forms of Plants and Statistical Plant Geography. Oxford University Press: London, Royaume Uni; $634 \mathrm{p}$.

Savard JP, Clergeau P, Mennechez G. 2000. Biodiversity concepts and urban ecosystems. Landscape and Urban Planning, $\quad$ 48: 131-142. DOI: 10.1016/S0169-2046(00)00037-2

Schwartz D, Dechamps R, Elenga H, Lanfranchi R, Mariotti A, Vincens A. 1995. Les savanes du Congo : une végétation spécifique de l'holocène supérieur. In 2è symposium de palynologie africaine, Tervuren (Belgique), CIFEG ; 99-108.
Sinsin B. 2001. Formes de vie et diversité spécifique des associations de forêts claires du nord du Benin. Syst. Geogr. Pl., 71 : 873-888. DOI: $10.2307 / 3668725$

Tiokeng B, Mapongmetsem PM, Nguetsop VF, Tacham WN. 2015. Biodiversité floristique et régénération naturelle sur les Hautes Terre de Lebialem (Ouest Cameroun). Int. J. Biol. Chem. Sci., 9(1): 56-68. DOI: 10.4314/ijbcs.v9i1.6

Troupin G. 1966. Étude phytocénologique du Parc national de l'Akagera et RwandaOriental: recherche d'une méthode d'analyse appropriée à la végétation d'Afrique intertropicale. h. d'Agr. Ens. Sup. Univ., Liège, 293 p.

Tuo FN, Koffi KJ, Kouassi AF, Kone M, Adama B, Bogaer J. 2017. Étude de la diversité, de l'endémisme et de la distribution spatiale des Rubiaceae de Côte d'Ivoire. Int. J. Biol. Chem. Sci., 11(2): 777-797. DOI: $10.4314 /$ ijbcs.v11i2.20

Vennetier P. 1977. Atlas du Congo ; Ed. Jeune Afrique : Paris ; $32 \mathrm{p}$.

Wezel A, Schlecht E. 2004. Inter-annual variation of species composition of fallow vegetation in semi-arid Niger. Journal of Arid Environments, 56(2): 265-282. DOI: https://doi.org/10.1016/S01401963(03)00049-1

White F. 1983. The Vegetation of Africa. A Descriptive Memoir to Accompany the UNESCO/AETFAT/UNSO Vegetation Map of Africa. Natural Resources Research, 20, UNESCO: Paris.

Yoka J, Loumeto JJ, Vouidibio J, Amiaud B, Epron D. 2010. Influence du sol sur la répartition et la production de phytomasse de savanes de la Cuvette Congolaise (République du Congo) Geo-Eco-Trop., 34 : 63 - 74. 